\title{
Reliability of Linear and Curvilinear Measurements on Cone- Beam Computed Tomography Images for the Evaluation of Implant Sites and Jaw Pathologies
}

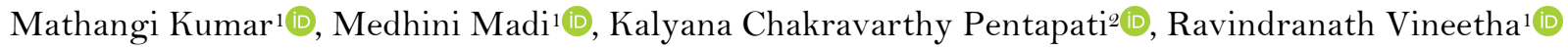

\footnotetext{
${ }^{1}$ Department of Oral Medicine and Radiology, Manipal College of Dental Sciences, Manipal, Manipal Academy of Higher Education, Manipal, Karnataka, India.

${ }^{2}$ Department of Public Health Dentistry, Manipal College of Dental Sciences, Manipal, Manipal Academy of Higher Education, Manipal, Karnataka, India.
}

Correspondence: Medhini Madi, Department of Oral Medicine \& Radiology, Manipal College of Dental Sciences, Manipal, Manipal Academy of Higher Education, Manipal, 576104, Karnataka, India. E-mail: medhini.madi@gmail.com

Academic Editor: Alidianne Fábia Cabral Cavalcanti

Received: 09 June 2020 / Review: 23 July 2020 / Accepted: 30 August 2020

How to cite: Kumar M, Madi M, Pentapati KC, Vineetha R. Reliability of linear and curvilinear measurements on conebeam computed tomography images for the evaluation of implant sites and jaw pathologies. Pesqui Bras Odontopediatria Clín Integr. 202 1; 21 :e0125. https://doi.org/10.1590/pboci.2021.023

\begin{abstract}
Objective: To evaluate the intra-examiner and inter-examiner reliability of linear and curvilinear measurements for the complete assessment of implant sites and jaw pathologies using Cone-Beam Computed Tomography (CBCT). Material and Methods: Fifty cone-beam computed tomographic images of patients were retrieved from the archives of Dentomaxillofacial Radiology. CBCT images taken for implant planning and evaluation of intrabony jaw pathologies (benign cyst/tumor) were included. Two expert oral and maxillofacial radiologists analyzed the images independently and made the measurements. The images for implant planning were analyzed for width, the height of the edentulous site, and the qualitative analysis of bone in the region. Jaw pathologies were assessed for linear dimensions and curvilinear measurements. Results: The inter-observer measurement error for implant site analysis ranged from 0.12 to $0.42 \mathrm{~mm}$ with almost perfect agreement (ICC: 0.94 to 1). The inter-observer measurement error for jaw pathology was 0.09 to $0.25 \mathrm{~mm}$ (ICC: 0.98-1). Curvilinear measurements showed perfect agreement between the observers. The intraobserver reliability for the various parameters used for the assessment of the implant site and jaw pathologies indicated almost perfect agreement. Conclusion: Reliability between the radiologists is high for various measurements on CBCT images taken for implant planning and jaw pathologies.
\end{abstract}

Keywords: Dental Implantation; Jaw, Edentulous; Diagnostic Imaging; Pathology, Oral. 


\section{Introduction}

Cone-beam computed tomography (CBCT) imaging provides detailed information by providing a three-dimensional view for the assessment of lesions affecting the maxillofacial region [1]. Measurements made on CBCT images play an important role in clinical situations. These include the evaluation of edentulous sites for implant placement, extent and size of cysts, tumors that affect the jaws, measuring airway spaces, and cephalometric analysis. CBCT software enables the operator to measure the linear and curvilinear dimensions of structures with a high degree of reliability $[2,3]$.

These measurements serve as a guiding tool for the clinicians to make appropriate decisions. It is well established that measurements of edentulous space for implant placement are accurately obtained via CBCT imaging [4-6]. Linear measurements on CBCT images are made for assessing the bone height and width for implant placement along with their distances from adjacent vital structures. These measurements are also used for predicting the dimensions of jaw pathologies and envisage their effect on the surrounding structures [7]. Other indications for measurement include pre-implant bone-density assessment, proximity to inferior alveolar nerve canal, and vital structures. However, the accurate reproducibility and reliability of such measurements become crucial in clinical decision-making and appropriate treatment planning.

It ensures consistency in the diagnosis between radiologists and clinicians and helps in the follow-up of patients. Thus, in these situations, the interobserver and intra-observer variations of these measurements become vital. Studies testing the reliability of these measurements have mostly been done on in vitro models $[4,8,9]$. Literature focussing on the reliability of these measurements using different software is also tested $[10,11]$. However, studies focusing on the reliability of these measurements on patients' CBCT images are very few [2].

Hence, the purpose of this study was to evaluate the intra-examiner and inter-examiner reliability of linear and curvilinear measurements on CBCT images taken for implant site analysis and assessment of jaw pathologies.

\section{Material and Methods}

Study Design and Sample

This retrospective, observational, cross-sectional study was conducted on the CBCT images from the archives of Dentomaxillofacial Radiology of Manipal College of Dental Sciences, Manipal Academy of Higher Education, Manipal, India. These CBCT images were acquired from i-CAT 17-19 Imaging System (Imaging Sciences International LLC, USA), and the images were viewed and analyzed through the In Vivo Dental Anatomage software. Images taken to assess implant sites and estimate the extent of jaw pathologies were included in the study irrespective of their field of view. CBCT images with artifacts in the area of interest were excluded from the study.

\section{Data Collection}

Sample size estimation was done based on the acceptable reliability (ICC: 0.5) and expected reliability (ICC: 0.8 ) [12]. The sample size yielded to be a minimum of 22 at the power of $80 \%$ and an alpha of $5 \%$ for two observations per image. Two investigators (Oral and maxillofacial radiologists) determined the linear measurements of various implant sites and curvilinear measurements of pathologies on the CBCT images at two instances; at baseline (To) and repeated the measurements two weeks later (T1) to evaluate the intraexaminer error. The image layer on which the measurements would be made was selected by marking the 
points on the axial section. Subsequent cross-sectional and reconstructed panoramic images were obtained automatically. The cross-section thickness was set as $0.5 \mathrm{~mm}$, and the section interval was set as $1 \mathrm{~mm}$. Both the observers recorded all the measurements in a specially designed proforma.

Measurements for the analysis of edentulous sites for implant placement were performed in three dimensions: the buccolingual width $(\mathrm{mm})$, the height of the edentulous region $(\mathrm{mm})$, and the qualitative assessment of the bone in Hounsfield units (HU). These readings were done on the "Implant screen" of the CBCT software, and measurements were made on the sagittal sections (Figure 1). These measurements were made using a wired mouse to position the marking tool cursor in the software. The measurements for the width of the alveolar ridge was obtained by marking two points at the level of the alveolar crest from the buccal/labial cortex to the palatal/lingual cortex. The width of the edentulous site was marked at two levels; one at the crestal level (W1) and another at $3 \mathrm{~mm}$ apically (W2). The height of the edentulous site was made by marking a point on the superior most aspect to the point $3 \mathrm{~mm}$ away from the adjacent vital structures. Qualitative analysis of the bone was marked in Hounsfield units at the edentulous site. The values of HU and corresponding favorability of the site for implant placement were recorded according to the values suggested by Misch [13].

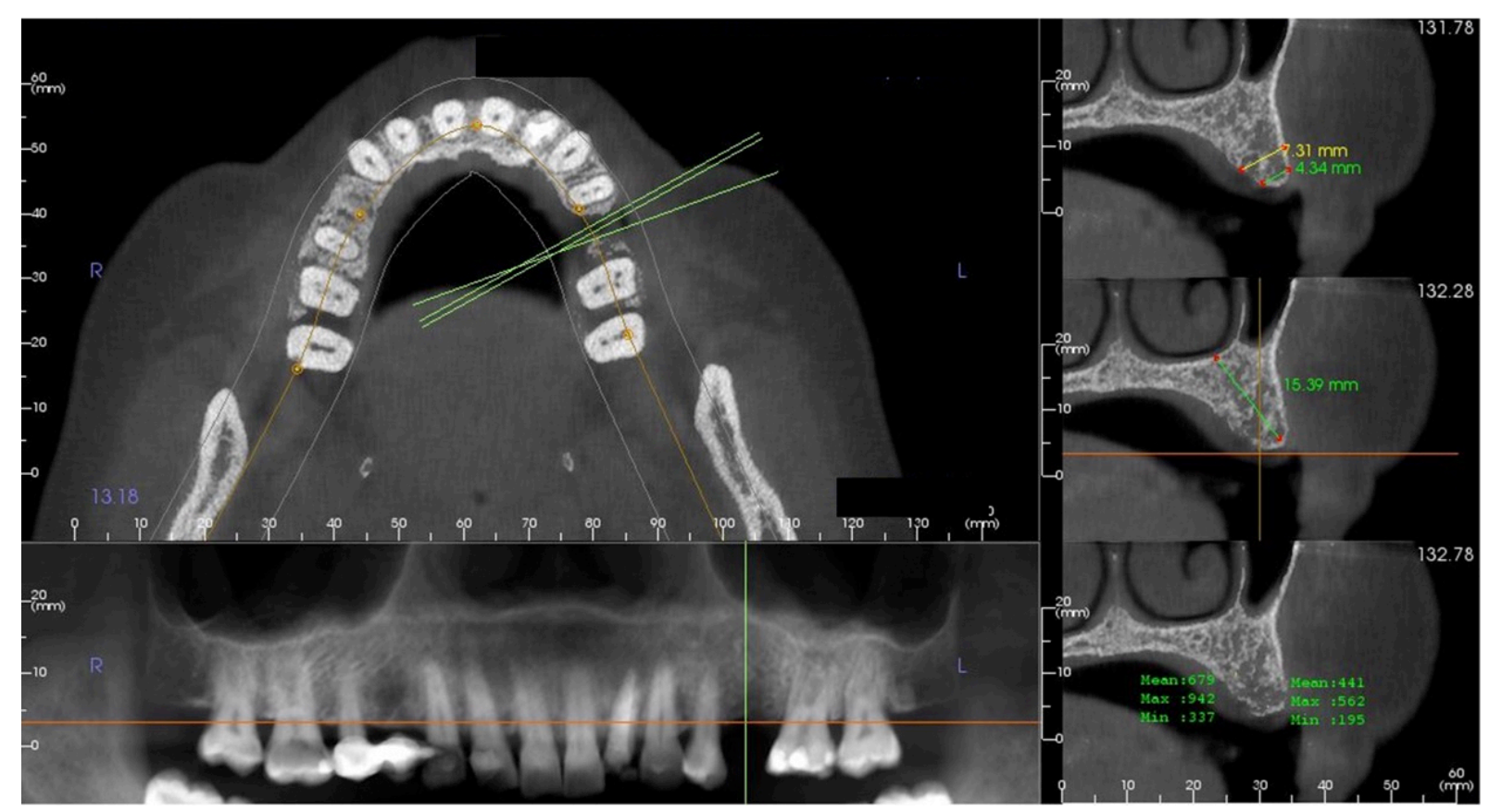

Figure 1. CBCT image showing the linear measurements and qualitative assessment of the edentulous site for implant placement.

Assessment of jaw pathologies was made on the axial and sagittal sections. The image layer on which the measurements were to be made was chosen by scrolling through the axial sections to obtain the specific section that depicts the jaw pathology in its maximum dimension. Curvilinear measurements were obtained by marking multiple points along the outline of the lesion in its maximum dimension (Figure 2). Then, the buccolingual, mesiodistal, superoinferior, and curvilinear measurements were made. Additionally, the effect of the pathology on the surrounding structures (root resorption, cortical perforation) was recorded as present or absent (Figure 3). 


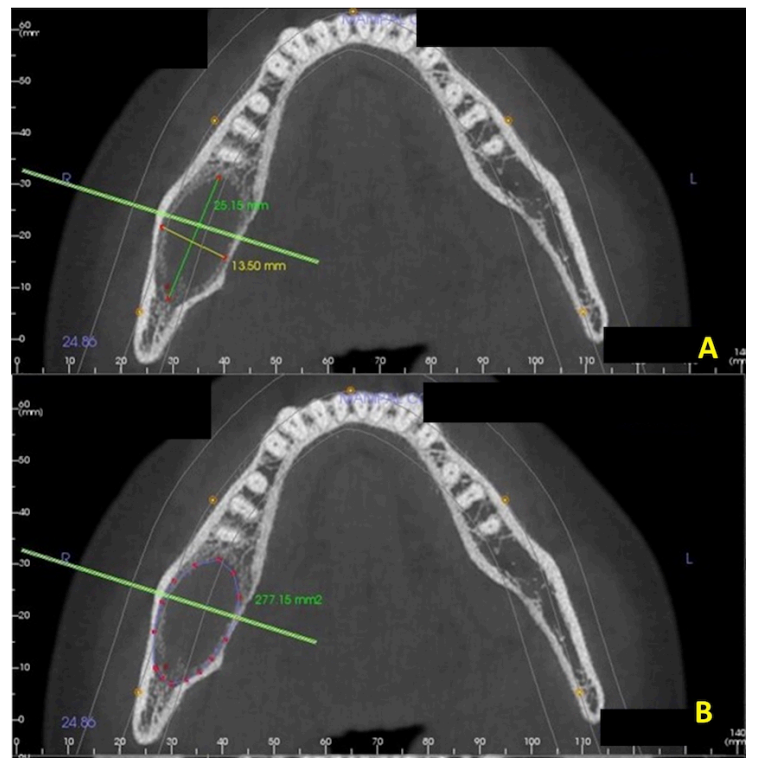

Figure 2 (A and B). CBCT image showing linear and curvilinear measurements made for cystic lesion in the posterior mandible.

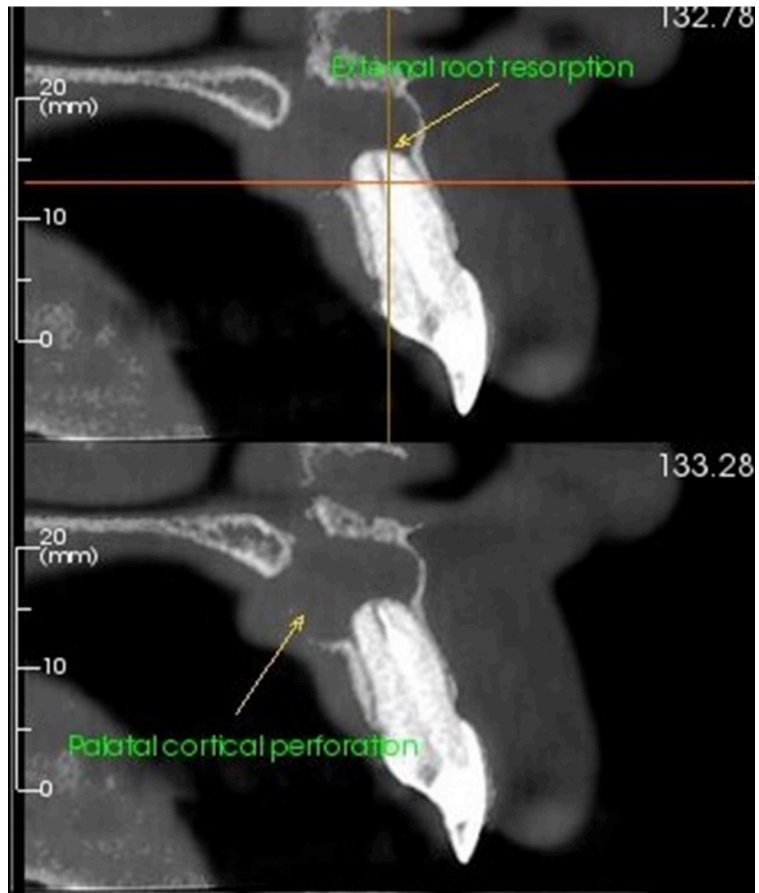

Figure 3. Sagittal sections of CBCT image showing the effect of periapical pathology on adjacent structures.

All the measurements were analyzed for inter and intraobserver reliability. The observers' measurement values were entered in the Microsoft Excel spreadsheet, and the agreement of the measurements made by the observers was compared using interclass correlation coefficients (ICC). Kappa statistics was applied to test the favorability of sites and effects on the surrounding structures.

Data Analysis 
Statistical analysis was performed using MedCalc Statistical Software version 14.8.1 (MedCalc Software bvba, Ostend, Belgium; https://www.medcalc.org). A two-way mixed model, with single measures and the absolute agreement, was done to calculate the intraclass correlation coefficient (ICC).

Ethical Aspects

Institutional Ethics Committee approved the conduct of the study (IEC 689/2019).

\section{Results}

This study was done on 50 CBCT images of patients whose images were analyzed for possible implant placement and assessment of the extent of jaw pathologies. Implant analysis and measurements were performed for 26 patients; bone pathologies of the jaws were assessed for 24 patients. Presurgical implant site analysis was done for 17 anterior edentulous sites of the maxillary arch and 9 posterior edentulous sites in the maxillary and mandibular posterior regions. Of the 24 bony pathologies, 12 belonged to the maxilla and 12 were in the mandibular region.

The mean measurements of the implant site assessment and jaw pathologies, along with the measurement error, are depicted in Table 1. The examiners' overall agreement was almost perfect, with an inter-examiner measurement error of $0.12 \mathrm{~mm}$ for the first dimension W1 (ICC=0.94). The agreement was strong even for the second reading W2 with an inter-examiner error of 0.17 (ICC=0.98). There was a perfect agreement with the second dimension (height of the edentulous ridge) with a measurement error of $0.42 \mathrm{~mm}$ and an ICC value of 1 (Table 2). There was substantial agreement between the observers for the third dimension, i.e., qualitative bone assessment values (Hounsfield units HU) and prediction of favourability of sites for implant placement showed (Kappa=0.62) (Table 3).

Table 1. Mean measurements and measurement error values of various parameters used for assessment of the implant sites and jaw pathologies.

\begin{tabular}{llccc}
\hline \multicolumn{1}{c}{ CBCT Analysis } & \multicolumn{1}{c}{ Parameter } & Mean Measurement (mm) & $\begin{array}{c}\text { Measurement } \\
\text { Error }\end{array}$ \\
& & Observer 1 & Observer 2 & \\
\hline Implant Site Assessment & Width of the bone at the alveolar crest (W1) & 6.75 & 6.87 & 0.12 \\
& Width of the bone 3 mm apical to W1 (W2) & 8.23 & 8.06 & 0.17 \\
& Height of the edentulous site & 15.17 & 14.75 & 0.42 \\
Jaw Pathology Assessment & Buccolingual Measurement & 11.73 & 11.83 & 0.10 \\
& Mesio Distal Measurement & 16.28 & 16.02 & 0.26 \\
& Supero Inferior Measurement & 11.79 & 11.88 & 0.09 \\
& Curvilinear Measurement & 56.35 & 55.58 & 0.77 \\
\hline
\end{tabular}

Table 2. Intra and Inter-observer reliability for various parameters used for assessment of the implant site.

\begin{tabular}{lcc}
\hline \multicolumn{1}{c}{ Measurements } & Inter-Observer ICC (95\% CI) & Intra-Observer ICC (95\% CI) \\
\hline Width of the bone at the alveolar crest (W1) & $0.94(0.87-0.97)$ & $0.99(0.99-1)^{\dagger}$ \\
Width of the bone few millimetres apical to W 1 (W2) & $0.98(0.96-0.99)$ & $0.99(0.99-1)^{\dagger}$ \\
& $1(0.99-1)^{+}$ \\
Height of the edentulous site & $1(0.99-1)$ & $1(0.99-1)^{\dagger}$ \\
& & $1(0.99-1)^{+}$ \\
\hline
\end{tabular}

†Observer 1; ${ }^{+}$Observer 2 . 
Table 3. Inter-observer reliability for favourability of implant site based on Hounsfield units.

\begin{tabular}{lccc}
\hline Favourability & \multicolumn{2}{c}{ Observer A } & Kappa \\
& Unfavourable & Favourable & \\
\hline Observer B & 11 & 3 & $0.615(0.31-0.92)$ \\
Unfavourable & 2 & 10 & \\
Favourable & & & \\
\hline
\end{tabular}

Assessment of jaw pathologies suggested that the various parameters made by the observers suggested almost perfect agreement (ICC values: 0.96 to 1) (Table 4). Additionally, there was a perfect agreement regarding the presence or absence of the effect of the jaw pathologies on surrounding structures $($ Kappa $=1)($ Table 5$)$.

The intraobserver reliability for the various parameters used for the assessment of the implant site and jaw pathologies indicated almost perfect agreement (Tables 2 and 4 ).

Table 4. Intra and Inter-observer reliability for various parameters used to assess jaw pathologies.

\begin{tabular}{|c|c|c|}
\hline Dimensions of the Pathology & Inter-Observer ICC $(95 \% \mathrm{CI})$ & Intra-Observer ICC (95\% CI) \\
\hline \multirow{2}{*}{ Buccolingual Measurements } & \multirow{2}{*}{$0.98(0.96-0.99)$} & $1(0.99-1)^{\dagger}$ \\
\hline & & $1(0.99-1) \ddagger$ \\
\hline \multirow{2}{*}{ Mesio Distal Measurements } & \multirow{2}{*}{$1(0.99-1)$} & $1(0.99-1)+$ \\
\hline & & $1(0.99-1)+$ \\
\hline \multirow{2}{*}{ Supero Inferior Measurements } & \multirow{2}{*}{$1(0.99-1)$} & $1(0.99-1)^{\dagger}$ \\
\hline & & $1(0.99-1) \ddagger$ \\
\hline \multirow{2}{*}{ Curvilinear Measurements } & \multirow{2}{*}{$1(0.99-1)$} & $1(1-1)^{+}$ \\
\hline & & $1(0.99-1)^{\ddagger}$ \\
\hline
\end{tabular}

Table 5. Inter-observer agreement on the effect of pathology on adjacent structures.

\begin{tabular}{cccc}
\hline Effect of Pathology on Adjacent Structures & \multicolumn{2}{c}{ Observer A } & Kappa \\
& Absent & Present & \\
\hline Observer B & & & \\
Absent & 23 & 0 & 1 \\
Present & 0 & 1 & \\
\hline
\end{tabular}

\section{Discussion}

CBCT has caused a paradigm shift from conventional planar imaging to $3 \mathrm{D}$ imaging in the field of maxillofacial radiology. CBCT plays a vital role in clinical situations like presurgical implant planning, assessment of intraosseous jaw pathologies, airway assessment, and cephalometric analysis in orthodontics [14]. Measurements made on the CBCT images are critical as they dictate treatment procedures, mainly; implant placement [5]. The measurements marked by the Oral and maxillofacial radiologist guides the operating clinician regarding the dimensions of intraosseous pathologies affecting the jaws.

Studies have evaluated the accuracy of CBCT measurements with actual clinical measurements in vitro scenarios and proved that CBCT values are highly reliable and accurate $[3,5,8,9,15,16]$. The measurements' reliability was excellent in studies on the diagnosis of upper airway disorders [17]. On the other hand, another study testing the reliability of CBCT in upper airway assessment showed that only oropharyngeal volume had excellent intra and interexaminer reliability and hypopharynx and nasopharynx volumes and the overall minimal cross-sectional area had a low agreement between the observers [18]. A systematic review on the reliability and reproducibility of three-dimensional cephalometric landmarks on CBCT showed that landmarks on the median sagittal line and dental landmarks had the highest reliability. In 
contrast, the landmarks on the condyle, porion, and orbitale presented lower levels of reliability [19]. Golfeshan et al. [20] evaluated the reliability of impacted maxillary canine feature measurements taken by CBCT and concluded that the angulation of the canine had the highest reliability, while the rotation and dilaceration had the lowest reliability.

Previous authors evaluated the reliability of linear measurements on CBCT for the proximity of mandibular third molars to the nerve canal. They found that there was a strong agreement between examiners with significant reliability and reproducibility [21]. A systematic review of the accuracy of measurements on $\mathrm{CBCT}$ images related to presurgical implant treatment planning suggested that $\mathrm{CBCT}$ can be considered an appropriate diagnostic tool for $3 \mathrm{D}$ preoperative planning. Also, it suggested that in clinical practice, the accuracy of measurements on $\mathrm{CBCT}$ images is influenced by factors such as patient movement, metallic artifacts, device-specific exposure parameters, and the software used [22].

An in vitro CBCT study utilizing different exposure parameters to test the accuracy of linear measurements around dental implants placed in the maxilla of dry human skulls showed acceptable accuracy for linear measurements around dental implants, despite the exposure parameters used [5]. Prashanti et al. [23] compared the measurements of implant site planning in 37 patients on ridge mapping, panoramic imaging, $\mathrm{CBCT}$ and found a moderate correlation between the measured bone widths from ridge mapping and CBCT. There was a high correlation in the measurements obtained between panoramic imaging and CBCT. On the other hand, Luk et al. [24] compared ridge mapping and CT for implant planning of twenty-one sites in 14 patients and concluded that the values differed significantly.

In the present study, the overall agreement between the examiners was almost perfect for all three dimensions. The inter-examiner measurement error was $0.12 \mathrm{~mm}$ for the first width (W1) and $0.17 \mathrm{~mm}$ for W2. Also, the inter-examiner measurement error for the height of the edentulous ridge was $0.42 \mathrm{~mm}$. The values observed from this study showed almost perfect agreement between the observers when compared to the findings from the study by Safi et al. [2]. They assessed the reliability of linear measurements of the alveolar ridges made by radiologists and periodontists on $23 \mathrm{CBCT}$ images and stated that there was almost perfect agreement between the observers.

Schnutenhaus et al. [25] performed a clinical study to evaluate the intrapersonal and interpersonal errors in bone structure determination on $\mathrm{CBCT}$ of 20 patients who underwent maxillary tooth extraction using a semi-automated procedure and concluded that all deviations in the values between the observers were within the range of the selected resolution of the $\mathrm{CBCT}$ device. The intrapersonal evaluation across both investigators resulted in an average deviation of $0.18 \mathrm{~mm}$ and the interpersonal analysis resulted in an average deviation of 0.15 and 0.26 at two different points [25].

CBCT imaging provides information on dental and maxillofacial structures for diagnosis and surgical planning in a multiplane mode (axial, coronal, and sagittal planes). It additionally offers important findings regarding the extent of the lesion, bone resorption, sclerosis of neighbouring bone, cortical expansion, presence of calcifications, and the proximity to other vital anatomical structures [1,14,26].

There are no studies that have tested the reliability of measurements of bone pathologies of the jaws on CBCT images. Also, this is the first-ever study that has tested the reliability of curvilinear measurements on CBCT images. These measurements are of significance to the surgeon for treatment planning and management. In the present study, we analyzed the reliability of linear, curvilinear measurements and the effect on surrounding structures of the cystic lesions and benign tumors of the jaws of 24 patients. There was almost perfect agreement between the examiners for these linear measurements (ICC: 0.98 to 1). The inter- 
examiner measurement error ranged from 0.09 to $0.25 \mathrm{~mm}$. Additionally, curvilinear measurements of these lesions also showed almost perfect agreement $(\mathrm{ICC}=1)$. The effect of the pathologies on the surrounding structures also showed perfect agreement (Kappa $=1)$.

Intrabony benign cystic and solid lesions are appropriately and accurately measured on CBCT imaging. The linear and curvilinear measurements of pathologies are of interest to the operating surgeon to visualize the possibility of pathological fracture due to the intrabony pathology. Additionally, these values provide a rough estimate of the amount of graft material required, planning for bone plating and reconstruction, and the amount of platelet-rich fibrin (PRF) solution that would be necessary. These preoperative measurements may serve as baseline values and help assess regression in the lesion's size during follow-up appointments of patients.

\section{Conclusion}

Hence, this study showed that there was almost perfect inter and intraexaminer reliability in the linear and curvilinear measurements made by the radiologists for implant site analysis and assessment of intrabony jaw pathologies. Minimal measurement differences may be attributed to reasons like the radiologist's skill, changes in the inclination at which measurements are made, and selection of the exact image layer by both the examiners. Future studies should assess the reliability of the measurements on CBCT in various other clinical scenarios.

\section{Authors' Contributions

\begin{tabular}{|c|c|c|}
\hline MK & https://orcid.org/0000-0003-1245-2314 & $\begin{array}{l}\text { Conceptualization, Methodology, Validation, Formal Analysis, Investigation, Resources, Data } \\
\text { Curation, Writing - Original Draft, Writing - Review and Editing, Visualization, Supervision } \\
\text { and Project Administration. }\end{array}$ \\
\hline MM & https://orcid.org/0000-0002-5484-5960 & $\begin{array}{l}\text { Conceptualization, Methodology, Validation, Formal Analysis, Investigation, Resources, Data } \\
\text { Curation, Writing - Review and Editing, Visualization, Supervision, Project Administration. }\end{array}$ \\
\hline $\mathrm{CP}$ & .org/0000-0002-54.62-5677 & Conceptualization, Software, Writing - Review and Editing and Supervision. \\
\hline $\mathrm{V}$ & https://orcid.org/0000-0002-8932-9982 & Conceptualization, Software, Writing - Review and Editing and Supervision. \\
\hline
\end{tabular}

\section{Financial Support}

None.

\section{Conflict of Interest}

The authors declare no conflicts of interest.

\section{Data Availability}

The data used to support the findings of this study can be made available upon request to the corresponding author.

\section{References}

[1] Pinsky HM, Dyda S, Pinsky RW, Misch KA, Sarment DP. Accuracy of three-dimensional measurements using conebeam CT. Dentomaxillofac Radiol 2006; 35(6):410-6. https://doi.org/10.1259/dmfr/20987648

[2] Safi Y, Kadkhodazadeh M, Esmaeil Nejad A, Vasegh Z, Amid R, Khalighi HR, et al. Reliability and reproducibility of linear measurements of alveolar ridges using cone-beam CT made by radiologists and periodontists. J Periodontol Implant Dent 2018; 7(2):35-9. https://doi.org/10.15171/jpid.2015.007

[3] Abdinian M, Baninajarian H. The accuracy of linear and angular measurements in the different regions of the jaw in cone-beam computed tomography views. Dent Hypotheses 2017; 8(4):100-3. https://doi.org/10.4103/denthyp.denthyp_29_17

[4] Al-Ekrish AA, Ekram M. A comparative study of the accuracy and reliability of multidetector computed tomography and cone beam computed tomography in the assessment of dental implant site dimensions. Dentomaxillofacial Radiol 2011; 4O(2):67-75. https://doi.org/10.1259/dmfr/27546065 
[5] Bohner LOL, Tortamano P, Marotti J. Accuracy of linear measurements around dental implants by means of cone beam computed tomography with different exposure parameters. Dentomaxillofac Radiol 2017; 46(5):20160377. https://doi.org/10.1259/dmfr.20160377

[6] Suomalainen A, Vehmas T, Kortesniemi M, Robinson S, Peltola J. Accuracy of linear measurements using dental cone beam and conventional multislice computed tomography. Dentomaxillofac Radiol 2008; 37(1):10-7. https://doi.org/10.1259/dmfr/14140281

[7] Weiss R 2nd, Read-Fuller A. Cone beam computed tomography in oral and maxillofacial surgery: an evidence-based review. Dent J 2019; 7(2):52. https://doi.org/doi:10.3390/dj7020052

[8] Baumgaertel S, Palomo JM, Palomo L, Hans MG. Reliability and accuracy of cone-beam computed tomography dental measurements. Am J Orthod Dentofac Orthop 2009; 136(1):19-25. https://doi.org/10.1016/j.ajodo.2007.09.016

[9] Menezes CC, Janson G, Massaro CDS, Cambiaghi L, Garib DG. Precision, reproducibility, and accuracy of bone crest level measurements of CBCT cross sections using different resolutions. Angle Orthod 2016; 86(4):535-42. https://doi.org/10.2319/040115-214.1

[10] Aksoy S, Kelahmet U, Hincal E, Oz U, Orhan K. Comparison of linear and angular measurements in CBCT scans using $2 \mathrm{D}$ and 3D rendering software. Biotechnol Biotechnol Equip 2016; 30(4):777-84. https://doi.org/10.1080/13102818.2016.1174077

[11] Abramovitch K, Rice DD, Christiansen EL, Sabeti M. Reproducibility of linear alveolar ridge measurements in 2 dicom CBCT viewing software programs: a pilot study. Oral Surg Oral Med Oral Pathol Oral Radiol 2019; $127(1): 31$. https://doi.org/10.1016/j.oooo.2018.07.012

[12] Walter SD, Eliasziw M, Donner A. Sample size and optimal designs for reliability studies. Stat Med 1998; 17(1):10110 .

[13] Misch CE, Judy KW. Classification of partially edentulous arches for implant dentistry. Int J Oral Implantol 1987; $4(2): 7-13$

[14] Shaabaninejad H, Sari AA, Rafiei S, Sari AM, Safi Y. The efficacy of CBCT for diagnosis and treatment of oral and maxillofacial disorders: a systematic review. J Islam Dent Assoc Iran 2014; 26(1):64-74.

[15] Timock AM, Cook V, McDonald T, Leo MC, Crowe J, Benninger BL, et al. Accuracy and reliability of buccal bone height and thickness measurements from cone-beam computed tomography imaging. Am J Orthod Dentofac Orthop 2011; 140(5):734-44. https://doi.org/10.1016/j.ajodo.2011.06.021

[16] Tolentino ES, Yamashita FC, de Albuquerque S, Walewski LA, Iwaki LCV, Takeshita WM, et al. Reliability and accuracy of linear measurements in cone-beam computed tomography using different software programs and voxel sizes. J Conserv Dent 2018; 21(6):607-12. https://doi.org/10.4103/JCD.JCD_314_18

[17] de Souza KRS, Oltramari-Navarro PVP, Navarro RL, Conti ACCF, de Almeida MR. Reliability of a method to conduct upper airway analysis in cone-beam computed tomography. Braz Oral Res 2013; 27(1):48-54. https://doi.org/10.1590/S1806-83242013000100009

[18] Zimmerman JN, Vora SR, Pliska BT. Reliability of upper airway assessment using CBCT. Eur J Orthod 2019; 41(1):101-8. https://doi.org/10.1093/ejo/cjy058

[19] De Oliveira Lisboa C, Masterson D, Motta AFJ, Motta AT. Reliability and reproducibility of three-dimensional cephalometric landmarks using CBCT: a systematic review. J Appl Oral Sci 2015; 23(2):112-9. https://doi.org/10.1590/1678-775720140336

[20] Golfeshan F, Miresmaili AF, Mosaddad SA. Reliability of impacted maxillary canine feature measurement obtained by CBCT. Ann Dent Spec 2018; 6(2):118-23.

[21] Santos TDS, Gomes AC, De Melo D, Melo A, Cavalcante JR, De Araújo LC, et al. Evaluation of reliability and reproducibility of linear measurements of cone-beam-computed tomography. Indian J Dent Res 2012; 23(4):473-8. https://doi.org/10.4103/0970-9290.104952

[22] Fokas G, Vaughn VM, Scarfe WC, Bornstein MM. Accuracy of linear measurements on CBCT images related to presurgical implant treatment planning: a systematic review. Clin Oral Implants Res 2018; 29 Suppl 16:393-415. https://doi.org/10.1111/clr.13142

[23] Prashanti E, Vynne OJ, Annishka A, Fickry FSS, Naurah MA, Idiculla J, et al. A comparative cross-sectional study of pre-implant site assessment using ridge mapping and orthopantomography (OPG) with cone beam computed tomography (CBCT). Res J Pharm Biol Chem Sci 2016; 7(5):1 185-92.

[24] Luk LC, Pow EH, Li TK, Chow TW. Comparison of ridge mapping and cone beam computed tomography for planning dental implant therapy. Int J Oral Maxillofac Implants 2011; 26(1):70-4.

[25] Schnutenhaus S, Graf M, Doering I, Luthardt RG, Rudolph H. Reproducibility of CBCT image analysis: a clinical study on intrapersonal and interpersonal errors in bone structure determination. Oral Radiol 2019; 35(2):152-8. https://doi.org/10.1007/s11282-018-0340-1

[26] Kumar M, Shanavas M, Sidappa A, Kiran M. Cone beam computed tomography - know its secrets. J Int Oral Health $2015 ; 7(2): 64-8$. 